\title{
A sufficient condition for a set of calibrated surfaces to be area-minimizing under diffeomorphisms
}

\author{
Doan The Hieu* \\ Department of Mathematics \\ College of Education, Hue University \\ 32 Le Loi, Hue, Viet Nam \\ dthehieu@yahoo.com; deltic@dng.vnn.vn
}

November 24, 2018

\begin{abstract}
We extend Choe's idea in Ch to nonpolyhedral calibrated surfaces and give some examples of polyhedral sets over right prisms and nonpolyhedral calibrated surfaces.
\end{abstract}

\section{Introduction}

In [Ch, Choe proved "Every stationary polyhedral set is area-minimizing under diffeomophisms leaving the boundary fixed". In his proof, a system of differential forms and orientations (of faces) was chosen at each singular edge. In fact, the differential forms are calibrations that calibrate the faces at each singular edge and have the vanishing sum. We observe that, the suitable orientations of faces at each singular edge determine the same orientation on it whenever it lies on the boundary of faces.

By the above observation, we extend Choe's idea by proving a sufficient condition for certain sets of calibrated surfaces (including polyhedral sets) to be area-minimizing under diffeomophisms leaving the boundary fixed. This sufficient condition, when applies to polyhedral sets, is also necessary.

We give some more examples of polyhedral sets over right prisms and first examples of nonpolyhedral calibrated surfaces (2-dimensional ones with singular sets of dimension 1 in $\mathbb{R}^{4}$ ).

\footnotetext{
*This work was completed at SNU, with the support of Korean Foundation for Advanced Studies. The author was also supported in part by the National Basic Research Program in Natural Sciences, Vietnam.
} 
The author would like to thank the gracious host scholar, Professor HongJong Kim, for his thoughtfulness and assistance. Many thanks to Professor Jaigyoung Choe for his help, suggestions and the invitation talking this result at his seminar. This paper was written while the author was visiting RIMGARC, Department of Mathematics of the Seoul National University, Korea. We would like to thank that institution for its hospitality and generous support.

\section{The theorem}

We refer the readers to $\mathrm{Ch}$ for the definition of polyhedral sets.

Let $\left\{C_{i}\right\}_{i \in I}$ be a set of calibrated surfaces of dimension $m$ in $\mathbb{R}^{n}(m<n)$ and $\left\{w_{i}\right\}_{i \in I}$ be the set of correspondent calibrations. That means for each $i \in I, w_{i}$ calibrates $C_{i}$ with a suitable orientation. Note that if $\omega_{i}$ calibrates $C_{i}$, then $-\omega_{i}$ calibrates $C_{i}$ with opposite orientation. Depending on a chosen orientation on $C_{i}$ we have the corespondent calibration to be $\omega_{i}$ or $-\omega_{i}$.

Let $\Sigma \subset \mathbb{R}^{n}$ be a set satisfies the following conditions:

(i) $\Sigma \subset \cup_{i \in I} C_{i}$,

(ii) the set $E=\Sigma \cap\left(C_{i} \cap C_{j}\right)$ is of dimension $m-1$ for every $i, j \in I, i \neq j$.

We call each $F_{i}=\Sigma \cap C_{i}$ a face, each $E$ a singular edge, the union of all singular edges $E$ the singular set $S$, the closure of $\partial F_{i} \sim S$ the boundary edge of $\Sigma$ in $F_{i}$, the union $\cup_{i \in I}\left(\partial F_{i} \sim S\right)$ the boundary $\partial \Sigma$ of $\Sigma$.

$\Sigma$ is said to be area-minimizing under diffeomorphisms leaving the boundary fixed if

$$
\operatorname{Vol}(\Sigma) \leq \operatorname{Vol}(\varphi(\Sigma))
$$

for any diffeomorphism $\varphi$ of $\mathbb{R}^{n}$ leaving the boundary of $\Sigma$ fixed.

Suppose $\left\{E_{j}\right\}_{j \in J}$ is the set of all singular edges and $\left\{F_{i}\right\}_{i \in I}$ is the set of all faces of $\Sigma$. Denote

$$
\begin{aligned}
& I_{E_{j}}=\left\{i: F_{i} \supset E_{j}\right\} \subset I, \\
& J_{F_{i}}=\left\{j: E_{j} \subset F_{i}\right\} \subset J .
\end{aligned}
$$

Theorem 2.1 Let $\Sigma$ be a set defined as above. Suppose that every singular edge $E_{j}$ lies on the boundary $\partial F_{i}, \forall i \in I_{E_{j}}$ and for each $E_{j}$ we can choose suitable orientations on $F_{i}, \forall i \in I_{E_{j}}$, such that:

(i) the orientations on $F_{i}, \forall i \in I_{E_{j}}$ determine the same orientation on $E_{j}$,

(ii) the corespondent calibrations have vanishing sum.

Then $\Sigma$ is area-minimizing under diffeomorphisms leaving $\partial \Sigma$ fixed.

Proof. The reasonings of the proof are very similar as that of the main theorem in $\mathrm{Ch}$ with some little changes.

Let $\varphi$ be a diffeomorphism leaving $\partial \Sigma$ fixed and $\varphi_{t}$ be the homotopy from the identity to $\varphi$. Suppose $G_{j}$ is the $m$-dimensional smooth surface swept out by $\varphi_{t}\left(E_{j}\right)$ and $D_{i}$ is $(m+1)$-dimensional surface swept out by $\varphi_{t}\left(F_{i}\right)$. We have

$$
\partial D_{i}=F_{i} \cup \varphi\left(F_{i}\right) \cup_{j \in J_{F_{i}}} G_{j},
$$


and hence

$$
\int_{\partial D_{i}} w_{i}=\int_{F_{i}} w_{i}+\int_{\varphi\left(F_{i}\right)} w_{i}+\sum_{j \in J_{F_{i}}} \int_{G_{j}} w_{i} .
$$

Since $w_{i}$ is a calibration that calibrates $F_{i}$, we get the following inequality:

$$
\operatorname{Vol}\left(F_{i}\right) \leq \operatorname{Vol}\left(\varphi\left(F_{i}\right)\right)-\sum_{j \in J_{F_{i}}} \int_{G_{j}} w_{i},
$$

and finally

$$
\operatorname{Vol}(\Sigma) \leq \operatorname{Vol}(\varphi(\Sigma))-\sum_{j \in J} \sum_{i \in I_{E_{j}}} \int_{G_{j}} w_{i} .
$$

By virtue of the assumtions of the theorem, we can assume the orientations on $F_{i}, \forall i \in I_{E_{j}}$, determine the same orientation on $G_{j}$ and since $\sum_{i \in I_{E_{j}}} w_{i}=0$, the last term equals zero. The theorem is proved.

Corollary 2.2 Let $\Sigma$ be a polyhedral set. Then $\Sigma$ is area-minimizing under diffeomorphisms leaving $\partial \Sigma$ fixed if and only if $\Sigma$ satisfies the assumptions in the Theorem 2.1]

Proof. The sufficiency follows from the above theorem and the necessity follows from the proof of the main theorem in $\mathrm{Ch}$.

\section{$3 \quad$ Examples}

1. At Ken Brakke's homepage, http://www.susqu.edu/facstaff/b/brakke/ we can see eight nice polyhedral cones, that are made of flat sheets meeting along triple lines with an equal angle $120^{\circ}$. All of them are area-minimizing under diffeomophisms leaving the boundary fixed by virtue of Theorem 2.1] Figures 1 provide more three polyhedral sets over right prisms.

2. Below are examples of nonpolyhedral calibrated surfaces that is areaminimizing under diffeomophisms leaving the boundary fixed.

Let $\mathbb{C}^{2} \equiv \mathbb{R}^{4}$ be complex plane with the standard complex structure $J_{1}$, $J_{1} e_{1}=e_{3} ; J_{1} e_{2}=e_{4}$.

Let $R_{2}, R_{3}, \ldots, R_{n}$ be the rotations of angles $\alpha, 2 \alpha, \ldots,(n-1) \alpha$ about the plane $\left\{x_{3}=x_{4}=0\right\}$, respectively, where $\alpha$ satisfies the condition $n \alpha=2 \pi, n \in$ $\mathbb{N}$. And let $J_{2}, J_{3}, \ldots, J_{n}$ be $(n-1)$ complex structures on $\mathbb{R}^{4}$ induced by $R_{2}, R_{3}, \ldots, R_{n}$

$$
J_{i}\left(e_{1}\right)=R_{i}\left(e_{3}\right), \quad J_{i}\left(e_{2}\right)=R_{i}\left(e_{4}\right) ; \quad i=2,3, \ldots, n .
$$

Denote $w_{1}, w_{2}, \ldots, w_{n}$ the Kähler forms correspondent to $J_{1}, J_{2}, \ldots, J_{n}$. We can easily to see that:

$$
\sum_{i=1}^{n} w_{i}=0
$$



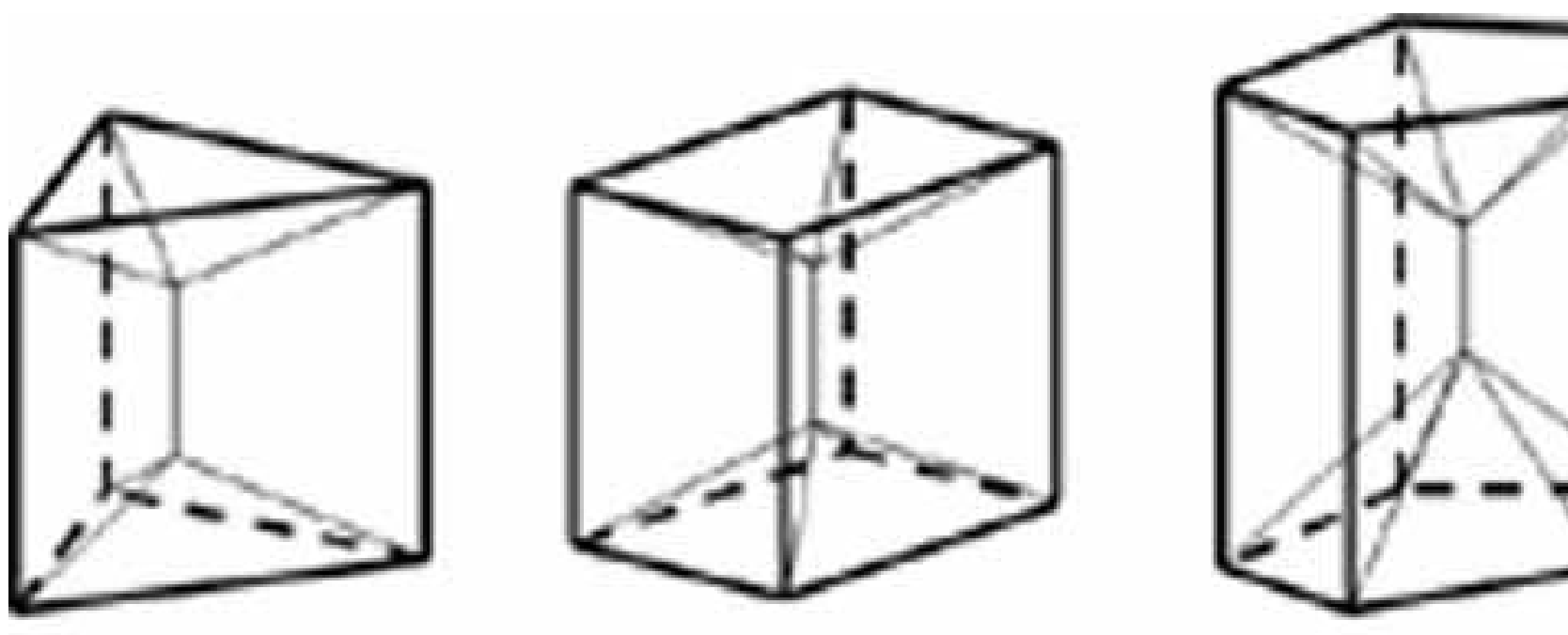

Figure 1: Polyhedral sets over right prisms.

Consider the complex curves:

$$
\begin{aligned}
C & =\left\{(z, w) \in \mathbb{C}^{2}: z=w^{2}\right\} \\
& =\left\{\left(x_{1}, x_{2}, x_{3}, x_{4}\right): x_{2}=x_{1}^{2}-x_{3}^{2} ; \quad x_{4}=2 x_{1} x_{3}\right\} .
\end{aligned}
$$

Let $D$ be the intersection of $C$ and $\left\{\sum_{i=1}^{4} x_{i}^{2}=1 ; x_{1} \geq 0 ; x_{3} \geq 0\right\}$. Note that $D$ contains two planar curves $\left\{x_{2}=x_{1}^{2} ; x_{3}=x_{4}=0 ; x_{1}^{2}+x_{2}^{2} \leq 1 ; x_{1} \geq 0\right\}$, and $\left\{x_{2}=-x_{3}^{2} ; x_{1}=x_{4}=0 ; x_{2}^{2}+x_{3}^{2} \leq 1 ; x_{3} \geq 0\right\}$. By using the rotations of angles $k \alpha, k=1,2 \ldots, n-1$ about the plane $\left\{x_{3}=x_{4}=0\right\}$ we get the images $D_{i}(i=2,3, \ldots, n)$ of $D$. Obviously, $w_{1}$ calibrates $D$ and $w_{i}$ calibrates $D_{i}, i=2,3, \ldots n$.

The set $\Sigma=D \bigcup D_{i}$ contains one singular edge and is area-minimizing under diffeomophisms leaving the boundary fixed by virtue of Theorem 2.1

Similarly, by using the rotations of angles $k \beta, k=1,2 \ldots, m-1 ; m \beta=2 \pi$ about the plane $\left\{x_{1}=x_{4}=0\right\}$, we get the images $D_{j}^{\prime}(j=2,3, \ldots, m)$ of $D$ and the images $\Sigma_{j}(j=2,3, \ldots, m)$ of $\Sigma$.

The set $D \bigcup D_{i} \bigcup D_{j}^{\prime}$ contains two singular edges. The set $\Sigma^{\prime}=\Sigma \bigcup \Sigma_{j}$ contains many singular edges. By the same reasoning as above, they are also area-minimizing under diffeomophisms leaving the boundary fixed.

\section{References}

[Ch] J. Choe, Every stationary polyhedral set in $\mathbb{R}^{n}$ is area minimizing under diffeomorphisms, Pacific J. Math. 175 (1996), 439-446. 
[HL] R. Harvey and H. B. Lawson, Calibrated geometries, Acta Math., 104 (1982), 47-157. 